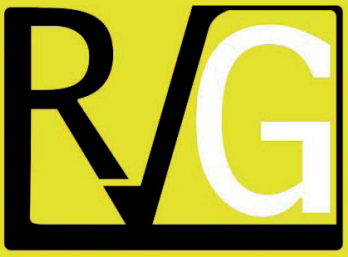

Julio - Septiembre, 2021

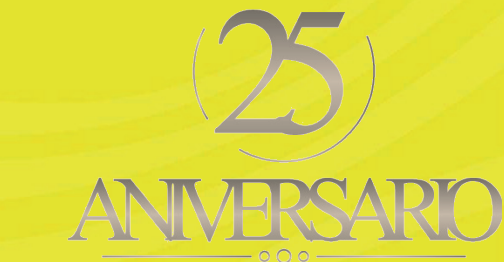

ANMERSARO
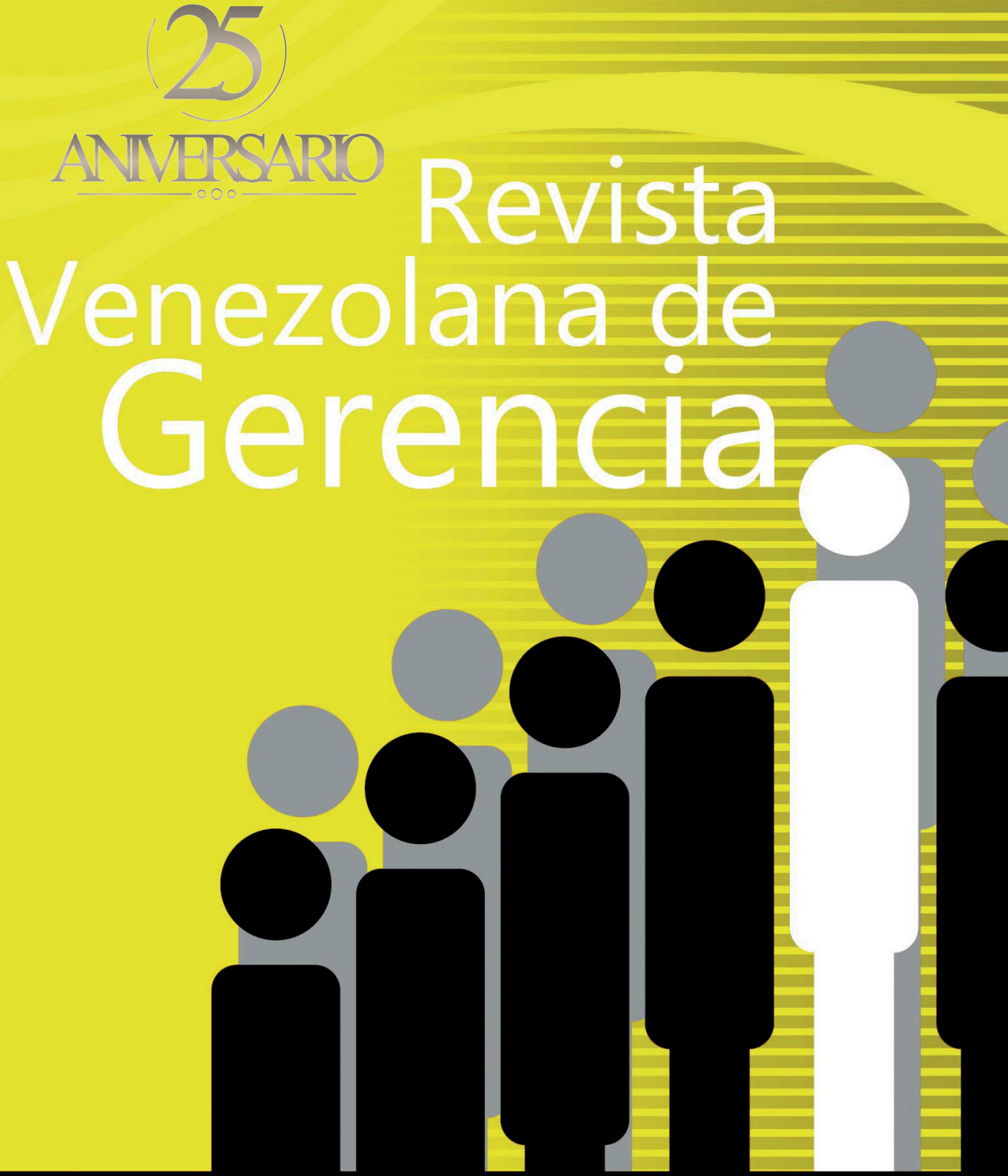

UNIVERSIDAD DEL ZULIA (LUZ)

Facultad de Ciencias Económicas y Sociales Centro de Estudios de la Empresa

ISSN 1315-99

Esta obra está bajo una licencia de Creative Comm Reconocimiento-NoComercial-Compartirlgual 3.0 Unpo http://creativecommons.org/licenses/by-nc-sa/3.0/deed.es 


\title{
Gestión del conocimiento en la innovación en pequeñas empresas de manufactura
}

\author{
Demuner Flores, María del Rosario*
}

\section{Resumen}

Gestionar el conocimiento es fundamental para innovar con éxito. En este sentido el objetivo fue analizar la gestión del conocimiento mediante la socialización, externalización, combinación e internalización, así como identificar posibles relaciones entre ellas y la innovación que desarrollan las pequeñas empresas de manufactura en México. A partir de una encuesta, con una aproximación cuantitativa, transversal y correlacional, se demostró que la socialización del conocimiento es la dimensión menos valorada y la internalización es la más alta. En el modelo multivariado que integra las cuatro dimensiones como proceso, la externalización a diferencia de las otras no es una variable predictora de la innovación. Se espera que las pequeñas y medianas empresas incrementen la conversión de su conocimiento explícito a tácito con el fin de lograr una innovación exitosa.

Palabras clave: conocimiento; gestión del conocimiento; innovación; pequeñas empresas; sector manufactura.

* Doctora en Ciencias Económico Administrativas, Maestra en Administración y Contador Público. Profesora Investigadora de la Facultad de Contaduría y Administración de la Universidad Autónoma del Estado de México. Miembro del Sistema Nacional de Investigadores del CONACYT. ORCID: http://orcid.org/0000-0002$\underline{4542-9113}$ 


\title{
Knowledge management for innovation purposes in small manufacturing companies
}

\begin{abstract}
Knowledge management is essential to innovate successfully. The purpose was to analyze knowledge management through socialization, externalization, combination and internalization, and to identify possible relationships among themselves and the innovation. A study with a quantitative, cross-sectional and correlational approach was carried out. A survey in small manufacturing companies in Mexico showed the socialization of knowledge is the least valued dimension and internalization is the highest. In the multivariate model with four dimensions as a process, externalization is not a predictor variable. SMEs are expected to increase the conversion of their tacit to explicit knowledge in order to achieve successful innovation.
\end{abstract}

Key Words: Knowledge; Knowledge management; Innovation; Small companies; Manufacturing.

\section{Introducción}

Las micro, pequeñas y medianas empresas en México, realizan importantes aportaciones a la economía. De acuerdo al censo 2018, las empresas ${ }^{1}$ de tamaño micro representan el $96.0 \%$ de las unidades económicas, las pequeñas $3.2 \%$ y las medianas $0.6 \%$; en cuanto a personal ocupado las empresas micro emplean el $35.0 \%$, las pequeñas $11.1 \%$ y las medianas $11.1 \%$; en cuanto a la producción bruta total, las empresas micro aportan $8.3 \%$, las pequeñas $6.5 \%$ y las medianas $11.8 \%$ (Instituto Nacional de Estadística y Geografía [INEGI], 2019: 20).

Sin embargo, su crecimiento se ve limitado por el cambio tecnológico, la complejidad para incorporar las innovaciones tecnológicas, poco apoyo gubernamental, dificultad para acceder al crédito, inadecuada infraestructura, baja especialización de los recursos humanos y falta de planeación (Demuner, 2011), aspectos importantes que obstaculizan la innovación y que han sido atribuidas al poco conocimiento que se gestiona al interior de la organización. $Y$ aunque algunas empresas gestionan el conocimiento en la práctica, éste no se da con una metodología, de forma más consiente, ni con mayor acierto (Arceo et al. 2021).

Las pequeñas y medianas empresas (PYME) tienen un vocabulario limitado sobre el conocimiento, un enfoque menos sistemático para 
apropiarse y compartir el conocimiento, y los beneficios percibidos de la gestión del conocimiento solo se orientan al mercado y dejan fuera la mejora de la eficiencia interna (Arceo et al. 2021).

Las PYMEs desconocen que la gestión del conocimiento (GC), a) impulsa la capacidad para responder rápidamente a clientes, creando nuevos mercados, desarrollando nuevos productos y dominando tecnologías nuevas; b) alimenta la confianza mutua entre el conocimiento de los trabajadores y administradores; c) facilita la cooperación en el manejo del tiempo en las tareas cotidianas; d) captura el conocimiento de los expertos y lo retiene para ayudar a la memoria de la organización (Restrepo et al. 2015).

La GC ha sido reconocida como un recurso estratégico que permite a las empresas esforzarse por captar y obtener provecho del conocimiento de todos sus integrantes a fin de tener mejoras continuas. Consiste en un proceso sistemático y específico para adquirir, organizar, mantener, aplicar, compartir y renovar el conocimiento tácito y explícito de todos los miembros de la organización, para aumentar el rendimiento y crear valor (Quiñonez y Rivera 2021; González y Álvarez, 2019; Pérez, 2016; Wang y Yang, 2016).

Por su aportación al desempeño empresarial, la GC ha sido estudiada a partir de su generación de rendimientos superiores (Bueno, 2005; García et al. 2019), éxito organizacional (Gutiérrez et al. 2015; Medina, 2017; Plaza, 2018) y valor agregado (Simancas et al. 2018; Wang y Yang, 2016). Así también se ha estudiado a partir de su relación con la innovación (Arceo et al. 2021; Fernández, 2012; Maldonado et al. 2012; Olea et al. 2016).

$$
\text { En esta línea teórica y }
$$

considerando la aportación de las PYME a la economía y en el afán de dar luz para resarcir sus debilidades fundamentadas en poca o nula GC, el objetivo de esta investigación es analizar la GC en sus cuatro dimensiones (socialización, externalización, combinación, internalización) e identificar si existe alguna relación entre las dimensiones de la GC y la innovación en pequeñas empresas.

La investigación se desarrolla en Toluca Estado de México. Este municipio es reconocido como el mejor del país con mayor valor agregado censal bruto en el sector de manufactura, pues encabeza la lista con el $3.1 \%$. Al ser la capital del Estado de México y debido a su importancia industrial, ocupa el tercer lugar en participación económica a la entidad (9.94\%) (Índice Municipal de Actividad Económica [IMAE], 2019), contribuye $10.9 \%$ en la generación de empleo (INEGI, 2015); resalta el sector de manufactura por albergar 11 parques y zonas industriales, ocupados principalmente por empresas del sector automotriz. Toluca cuenta con el $64.6 \%$ de empresas de manufacturas de todo el Estado, donde las micro y pequeñas empresas representan el $97 \%$ (INEGI, 2015).

El trabajo se organiza, presentando en el primer apartado, el sustento teórico de la GC con fines de innovación; el segundo apartado se refiere el método empleado y los resultados obtenidos, y finaliza con las conclusiones de la investigación.

\section{Contextualización de la relación entre la innovación y la gestión del conocimiento}

La innovación y la gestión del 
Demuner Flores, María del Rosario

Gestión del conocimiento en la innovación en pequeñas empresas de manufactura

conocimiento van de la mano, por ello, se plantean elementos esenciales asociados a dichos conceptos.

\subsection{Innovación}

La innovación es la concepción e implantación de cambios significativos en el producto, el proceso, el marketing o la organización de la empresa (Manual de Oslo, 2018). La innovación es un mecanismo de respuesta al ambiente que asegura la supervivencia y los recursos de la organización (Gopalakrishnan y Damanpour, 1997). La innovación es más común en las grandes empresas, su propensión a involucrarse se da como resultado de contar con recursos financieros y humanos; en cambio las PYMEs se encuentran en desventaja, en parte debido a limitaciones emanadas del tamaño.

Restrepo et al. (2015) en su estudio de micro, pequeñas y medianas empresas argumentan que no están involucradas en procesos de innovación debido a su bajo interés lo cual provoca reducir las posibilidades de competir exitosamente en los mercados tanto internos como externos. A pesar de ello, la contraparte argumenta que este grupo de empresas, debido a su tamaño, puede lograr un buen ambiente de trabajo, tener una menor complejidad de organización, mayor nivel de flexibilidad en sus procesos, además de generar comunicación más directa que motiva a los empleados e identificarse con los objetivos de la empresa y los beneficios de la innovación (Lloyd et al. 2002).

La posición que defiende este trabajo, es que el tamaño no es lo que dificulta la innovación en las PYME, es el desconocimiento de cómo hacerlo. Para que la innovación sea efectiva, requiere sistemas de gestión específicos y recreación de un ambiente propicio para adoptar o generar innovación para crear nuevos productos y mejorar los procesos de producción (De Jong y Vermeulen, 2006).

Un ambiente que fomenta la innovación se relaciona con la existencia de personal técnico especializado, científicos e ingenieros, que poseen conocimiento derivado de la experiencia y el desarrollo científico y técnico (Renuka y Venkateshwara, 2006). Además, la innovación debe soportarse en la tecnología utilizada en la empresa, en la capacidad de la organización para buscar soluciones más creativas a los problemas de la empresa, a la búsqueda y adquisición de conocimiento en el exterior y a los procesos que directamente promueven la innovación (Fernández, 2012).

Otra variable observada que favorece la innovación es el capital relacional. Las PYMEs insertas en cadenas productivas (Olea et al. 2016), por su condición de dependencia de grandes empresas y dada la atención casi personalizada, son beneficiadas por la derrama de conocimientos que involucra mejoras a los productos, procesos, tecnología, entre otros. La cercanía, hace que interactúen más fácilmente con sus clientes y proveedores y mejoren sus prácticas con las aportaciones de expertos y consultores (Olea et al. 2016).

En este contexto, se asume, que la innovación depende de la capacidad de absorción que la organización desarrolla a partir de adquirir, asimilar y explotar conocimiento nuevo del entorno y combinarlo con el conocimiento previamente gestado (Cohen $y$ Levinthal, 1990). Con estos argumentos, el trabajo pretende explorar a través de un modelo de GC, qué tanto este grupo de empresas acude al conocimiento con 
fines de innovación.

\subsection{Gestión del conocimiento}

La heterogeneidad entre empresas se asocia a las rutinas organizacionales, recursos tecnológicos para procesos innovadores y estrategias empresariales, que generalmente involucran la creación, coordinación y gestión de conocimientos que impactan el desempeño de las empresas (Arantes et al. 2021).

Existen factores incidentes en la GC, entre los que resaltan las personas, la tecnología y los procesos o rutinas internos implementados por la organización; los miembros comparten el conocimiento tácito que poseen para convertirlo en una ventaja que agrega valor a la organización (González y Álvarez, 2019). Estos factores constituyen la base del diseño e implementación de sistemas que buscan identificar, capturar y compartir el conocimiento de una organización para convertirlo en valor para sí misma (Pérez, 2016); su inclusión a un proceso sistemático y específico se da con el fin de cumplir los objetivos corporativos, aumentar el rendimiento y crear valor (Wang y Yang, 2016).

Desde esta concepción, la GC participa en un macroproceso corporativo soportado en procesos estratégicos, tomando como base un grupo de actividades que van desde la creación hasta la transmisión del conocimiento como activo de alto valor en pro del éxito organizacional (Medina, 2017).

La GC con fines de innovación se direcciona al éxito de la organización, al mejoramiento del desempeño y al logro de una ventaja competitiva. Por un lado, se distingue la GC como un proceso dinámico enfocado a la administración de los recursos y capacidades empresariales y como generador de resultados que se adapta al entorno en condiciones de incertidumbre que permite mejorar la posición competitiva $y$, por ende, la obtención de resultados superiores (Bueno, 2005).

También, se identifica que la GC se materializa en condiciones tanto internas como externas. Las condiciones internas (propósito estratégico, estructura flexible, tecnologías de información y comunicación, y ambiente) y las condiciones externas que se vislumbran en el entorno competitivo, responden al dinamismo, complejidad y rivalidad de la empresa y su entorno (Teece, 2016). La GC impulsa e influencia la capacidad innovadora, con el objetivo de mejorar el rendimiento empresarial (González y Álvarez, 2019) y lograr la ventaja competitiva (Porter, 1990).

Desde la postura de Nonaka y Takeuchi (1995) quienes defienden que la organización no puede crear valor sin la iniciativa de los integrantes de la organización y las interacciones que se establecen en los grupos de trabajo, se elige su modelo de GC Espiral de Conocimiento, porque postula que los saberes se crean cuando se produce una transformación del aprendizaje tácito de los individuos en explícito a nivel grupal y organizacional; cada uno de los miembros de tales colectivos lo interiorizan, convirtiéndolo de nuevo en tácito (Nonaka y Takeuchi,1995).

Los autores del modelo afirman que las compañías crean nuevo conocimiento e innovación no sólo procesando información del exterior al interior, sino haciéndolo fundamentalmente del interior al exterior. Se considera que la interacción entre ambos tipos de conocimiento constituye la dinámica central de la creación del conocimiento en las organizaciones, según la cual se 
pueden dar cuatro etapas de conversión:

1. Socialización: tácito a otro tácito. Es un proceso en el que un individuo transmite algún saber, comparte experiencias y pensamientos con otro individuo, quien incrementa su conocimiento y llega a conseguir niveles cercanos a los del emisor mediante la captación y diseminación de conocimientos y experiencias. Se recomienda interacción cara a cara mediante equipos autodirigidos, observación, imitación, discusiones detalladas, interacción con clientes (Demuner et al. 2015).

2. Externalización: de tácito a explícito. La interacción de los actores en ciertas actividades o procesos crea modelos mentales donde se comparten ideas, experiencias y conocimientos. Enseguida, los involucrados deben desarrollar una reflexión individual para luego generar una colectiva. Como resultado, se obtienen conceptos explícitos, que después deben validarse con el fin de comprobar que todos los involucrados los comprendan. Los medios sugeridos para externalizar el conocimiento, según Demuner et al. (2015) son: metáforas, hipótesis, analogías, modelos, slogans, diagramas, esquemas.

3. Combinación: de explícito a explícito.

Persigue capitalizar el conocimiento en toda la organización. Es la parte del proceso que sintetiza los conceptos explícitos y los traslada a una base de conocimiento, mediante la captura y la integración de nuevo conocimiento explícito esencial, a través de la recopilación, la reflexión y la síntesis. Primero, logra ordenar, agrupar y categorizar la información reunida; en segundo lugar esta información analizada, retroalimentada, enriquecida por todos los integrantes del equipo, se convierte en conocimiento que debe ser clasificado o contextualizado por funciones, áreas, departamentos, etc. En tercer lugar, se documenta el conocimiento que queda capitalizado y a disposición dentro de la organización. Finalmente se someten a revisión los documentos elaborados, planos, informes, minutas de juntas, conversaciones telefónicas o por redes sociales, manuales, políticas, reglas, minería, etc.

4. Internalización: de explícito a otro tácito. Es la etapa del proceso en la que se amplía el conocimiento tácito de los individuos a partir del conocimiento explícito de la organización al depurarse este último y convertirse en conocimiento propio de cada persona. La condición necesaria es que el conocimiento explícito sea vivido o experimentado, bien al pasar personalmente por la experiencia de realizar una actividad, bien a través de la participación, de las simulaciones, o de los ejercicios de juegos de rol, para que así el individuo lo haga propio según su estilo y sus hábitos particulares (Nonaka y Takeuchi, 1995). La espiral del conocimiento indica que estos saberes no son estáticos, pues siempre habrá algo más que aprender, se inicia otro ciclo.

Con el fin de realizar una aportación a las PYMEs, se explora en la presente investigación la siguiente hipótesis:

$\mathrm{H}_{1}$ : La socialización, la externalización, la combinación y la internalización se relacionan positiva y 
significativamente con la innovación de las pequeñas empresas manufactureras de Toluca, Estado de México.

\section{Consideraciones metodológicas de la investigación}

Dada la importancia que tienen las pequeñas empresas mexicanas en el sector manufactura a nivel nacional, estatal y municipal, este trabajo tuvo el objetivo de analizar la GC en sus cuatro dimensiones (socialización, externalización, combinación, internalización) e identificar alguna relación entre las dimensiones de la GC y la innovación en pequeñas empresas. Se realizó un estudio cuantitativo, transversal y correlacional mediante el diseño de un modelo multivariado que permitió la descripción de la correlación de las variables estudiadas.

La población en estudio emanó del Censo Económico 2019 (INEGI, 2019), donde el Estado de México albergó 61,840 unidades económicas de la industria manufacturera; donde 58,119 son empresas de tamaño micro, 2,237 son pequeñas, 974 son medianas y 510 son empresas grandes. En Toluca, de acuerdo al censo 2014 (INEGI, 2015) las pequeñas y medianas empresas manufactureras representaron el 3\%. La muestra no probabilística por intensión, se conformó por la respuesta de 136 empresas, número que alcanzó el $4 \%$ de respuesta respecto a las pequeñas y medianas empresas del Estado de México.

Para recabar la información se acudió al diseño de un cuestionario a través de la revisión de la literatura (Chauvet, 2014; Cohen y Levinthal, 1990 y 1994; Fernhaber y Patel, 2012; Flor et al. 2011; Nieto y Quevedo, 2005; Zahra y George, 2002).

La variable dependiente es Innovación que incluye preguntas relacionadas a la realización de investigaciones de mercado; presupuesto dirigido a actividades de investigación y desarrollo; uso de equipos de investigación; conocimiento sobre nuevas tecnologías empleadas por sus competidores; conocimiento de la posición de sus competidores respecto al desarrollo y lanzamiento de nuevos productos; identificación de su capacidad para desarrollo tecnológico; incentivos a sus empleados por actividades de lyD; proyectos de desarrollo para nuevos productos; esfuerzos destinados al desarrollo de nuevos productos. Las variables independientes son las cuatro dimensiones de la espiral de conocimiento (Nonaka y Takeuchi, 1995) (Cuadro 1). 


\section{Cuadro 1 \\ Variables independientes}

\begin{tabular}{|c|c|c|c|}
\hline Socialización & Externalización & Combinación & Internalización \\
\hline $\begin{array}{l}\text { Preguntas relacionadas } \\
\text { a búsqueda de } \\
\text { información sobre nuevos } \\
\text { productos, procesos, } \\
\text { tecnología novedosa con } \\
\text { proveedores e industrias } \\
\text { afines; búsqueda de } \\
\text { colaboración con otras } \\
\text { empresas para nuevos } \\
\text { desarrollos; concentración } \\
\text { de la información } \\
\text { obtenida de las diferentes } \\
\text { áreas; consensar } \\
\text { información e ideas } \\
\text { con los departamentos } \\
\text { involucrados en la } \\
\text { innovación; comunicación } \\
\text { con otros organismos para } \\
\text { identificar oportunidades } \\
\text { para introducir nuevos } \\
\text { productos; difusión de la } \\
\text { información con el resto } \\
\text { de la organización. }\end{array}$ & $\begin{array}{l}\text { Preguntas enfocadas } \\
\text { a la relación del } \\
\text { nuevo conocimiento } \\
\text { con lo que los } \\
\text { trabajadores ya saben } \\
\text { o han experimentado; } \\
\text { adaptación o imitación } \\
\text { de nuevas tecnologías } \\
\text { generadas por los } \\
\text { competidores; análisis } \\
\text { de información captada } \\
\text { a través de servicios de } \\
\text { consultoría; dinámicas } \\
\text { de retroalimentación } \\
\text { llevada a cabo } \\
\text { por equipos } \\
\text { multidisciplinarios; } \\
\text { supervisión de la } \\
\text { difusión de conocimiento } \\
\text { entre los diferentes } \\
\text { departamentos. }\end{array}$ & $\begin{array}{l}\text { Diseño de rutinas } \\
\text { para documentar } \\
\text { la información y } \\
\text { los desarrollos } \\
\text { tecnológicos; creación } \\
\text { de bases de datos, } \\
\text { manuales, instructivos, } \\
\text { etc. para usar en } \\
\text { nuevos desarrollos; } \\
\text { monitoreo del proceso } \\
\text { de introducción de } \\
\text { nuevos productos } \\
\text { al mercado para } \\
\text { su detección y } \\
\text { corrección de errores; } \\
\text { coordinación y } \\
\text { documentación de la } \\
\text { mejora de procesos } \\
\text { e innovaciones de } \\
\text { productos en las } \\
\text { distintas áreas de la } \\
\text { empresa; formalización } \\
\text { de la información } \\
\text { mediante minutas, } \\
\text { documentos, guías, } \\
\text { políticas, prototipos. }\end{array}$ & $\begin{array}{l}\text { Vivencias y experiencias } \\
\text { prácticas cobre la } \\
\text { incorporación de mejoras a } \\
\text { manuales, guías, prototipos } \\
\text { de los productos y procesos } \\
\text { con miras a innovación; } \\
\text { formular procedimientos y } \\
\text { estrategias que respondan } \\
\text { a acciones de los } \\
\text { competidores; formulación } \\
\text { de planes, presupuestos } \\
\text { y vigilancia del entorno; } \\
\text { puesta en práctica de } \\
\text { planes de marketing; } \\
\text { diseño de estrategias que } \\
\text { respondan a gustos y } \\
\text { preferencias de los clientes. }\end{array}$ \\
\hline
\end{tabular}

Fuente: elaboración propia en base a Chauvet (2014), Cohen y Levinthal (1990, 1994), Fernhaber y Patel (2012), Flor et al. (2011), Nieto y Quevedo (2005) y Zahra y George (2002).

La encuesta se realizó durante el primer semestre de 2019 a ejecutivos de primer nivel o propietarios de pequeñas empresas ubicadas en Toluca, inscritas en el Directorio Empresarial del Estado de México. El cuestionario se aplicó de forma auto administrada, con carácter anónimo. Se compuso por preguntas cerradas a calificarse con una escala tipo Likert de 5 puntos, de 1 (nunca) a 5 (siempre). Para el análisis de los datos, en primer lugar, se acudió al uso de regresiones simples y posteriormente, se definió un modelo de regresión múltiple, cuyos resultados se explican en los siguientes apartados.

\section{Gestión del conocimiento e innovación en pequeñas empresas manufactureras mexicanas: Resultados}

La muestra quedó conformada con 136 empresas manufactureras de tamaño pequeño. El $38 \%$ son empresas de giro textil, el $19 \%$ se dedican a la fabricación de alimentos y bebidas, 15\% son empresas de la industria automotriz, $11 \%$ fabricación de muebles, $8 \%$ productos artesanales y $9 \%$ otros.

Para visualizar el desarrollo de las dimensiones de la GC, en el diagrama 1 , se puede apreciar que la dimensión menos desarrollada es la socialización y la dimensión con valores más altos es la internalización. 


\section{Diagrama 1 \\ Presencia de las dimensiones de gestión del conocimiento.} Socialización

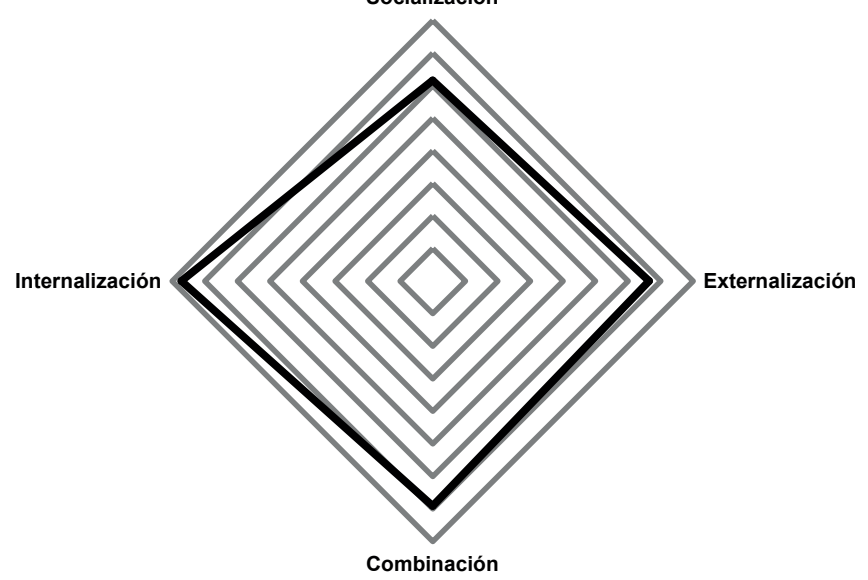

N:136 PYME. Medias: Socialización 30.8, externalización 33.2, combinación 34.5, internalización 38.6

Fuente: elaboración propia.

Para analizar la bondad de ajuste de los datos con la distribución de probabilidad normal se aplicó la prueba de Kolmogorov-Smirnov de una muestra (Tabla 1). Los resultados indican que todas las variables, exceptuando combinación ( $p=<0.05$ ), muestran una distribución normal a un nivel de significancia de 0.01, (Hair et al. 2010).

\section{Tabla 1}

\section{Prueba de normalidad de Kolmogorov-Smirnov $(n=136)$}

\begin{tabular}{lcc}
\hline Variable dependiente & Kolmogorov-Smirnov & Significancia \\
\hline Socialización & 0.101 & 0.002 \\
\hline Externalización & 0.101 & 0.002 \\
\hline Combinación & 0.082 & 0.027 \\
\hline Internalización & 0.126 & 0.000 \\
\hline Innovación & 0.096 & 0.003 \\
\hline
\end{tabular}

Fuente: elaboración propia

Para el análisis de confiabilidad o consistencia interna de la escala, se empleó la prueba alfa de Cronbach. Todos los casos mostraron valores superiores a 0.7 (Innovación 0.851, socialización 0.837 , externalización 0.777 , combinación 0.832 , internalización 0.724 ) por lo que la escala refleja un 
Demuner Flores, María del Rosario

Gestión del conocimiento en la innovación en pequeñas empresas de manufactura

buen nivel de confiabilidad (Hair et al. 2010).

Como se muestra en la Tabla 2, las diferentes dimensiones de la GC se

correlacionan significativamente con la variable innovación, los valores oscilan entre 0.577 y 0.824 , siendo todas significativas a nivel 0.001 .

\section{Tabla 2}

\section{Correlaciones Pearson}

\begin{tabular}{lcccc} 
& Externalización & Combinación & Internalización & Innovación \\
\hline Socialización & $0.710^{* *}$ & $0.687^{* *}$ & $0.577^{* *}$ & $0.824^{* *}$ \\
\hline Externalización & & $0.790^{* *}$ & $0.690^{* *}$ & $0.753^{* *}$ \\
\hline Combinación & & $0.738^{* *}$ & $0.775^{* *}$ \\
\hline Internalización & & & $0.724^{* *}$ \\
\hline
\end{tabular}

** La correlación es significativa al nivel 0.001 (bilateral).

Fuente: elaboración propia

Para analizar la relación de las dimensiones de la GC con la innovación se calculó un modelo de regresión lineal múltiple con el software IBM SPSS versión 21. Se decidió usar regresión lineal para especificar, estimar y evaluar modelos de relaciones entre el grupo de variables y en cierta medida usarlas con fines de predicción (Hair et al. 2010). Antes de calcular el modelo final, se calcularon regresiones simples.

$$
\text { Los criterios estadísticos }
$$
empleados que soportan la regresión inician con el uso del tamaño de la muestra. Ésta es mayor a 100 individuos, el número de casos es mayor a $50+4 \mathrm{~m}$, donde " $m$ " es el número de variables independientes, y la variable dependiente muestra una distribución normal (KS $=0.096, p=.003$ ), se considera que la muestra es suficiente para arrojar resultados confiables en el análisis de regresión planteado (Tabachnick y Fidell, 2013). Para descartar que el modelo propuesto calculado ofrezca interpretaciones erróneas sobre las relaciones entre las variables incluidas se probaron los siguientes supuestos.

Supuesto de independencia de los residuos. La prueba de Durbin-Watson corresponde a 1.601, al encontrarse en el parámetro permitido (1.5 y 2.5 ) señalado por Pardo y Ruiz (2002:375), se asume la independencia de los residuos.

Supuesto de no colinealidad. Aunque existen correlaciones significativas, la multicolinealidad de los datos es grave si por lo menos uno de los coeficientes de correlación simple, entre las variables independientes, es de al menos 0.6 (Tarling, 2009), lo que no ocurre entre ninguna de las variables que se incluyeron en el modelo.

El FIV del modelo fluctúa entre 2.210 y 3.491. De acuerdo con Bowerman et al. (2007) no existe multicolinealidad entre las variables independientes dado que el FIV más grande no es mayor que diez. Con respecto a los valores de tolerancia, éstos fluctuaron entre .0 .286 y 0.452. De acuerdo con Tarling (2009), si $1 / F I V=<1$ existe multicolinealidad, para este caso: $1 / 10=0.1$, los valores reportados son mayores, se acepta el 
supuesto de no multicolinealidad.

Como se observa en la Tabla 3 , el modelo simple reporta que cada una de las cuatro dimensiones de GC se relaciona significativa y positivamente con la variable dependiente. Todas las correlaciones lineales simples entre variables resultaron ser mayores a 0.692 con $p<0.001$, lo que permite la capacidad predictiva de un modelo de regresión lineal a partir de ellas (Tarling, 2009).

Así mismo, en la Tabla 3 se presenta el modelo multivariado que mejor ajusta a la hipótesis planteada. El modelo explica el $79.3 \%$ de la varianza en el uso de la GC con fines de innovación $\left(R^{2}=0.793\right)$, con un error estándar de 0.365 . Este error estándar total es menor al número de parámetros del modelo (obtenidos por $\mathrm{k}+1$, donde k es el número de variables regresoras); por lo tanto, es posible asumir que el sesgo del modelo es pequeño (Milton y Arnold, 2004). La prueba $F$ del modelo es de 125.369 ( $p=0.000)$.

De acuerdo con los resultados de este análisis de regresión múltiple, la hipótesis $H_{1}$ puede ser aceptada solo parcialmente. Socialización $(\AA)=.414$, $\mathrm{t}=8.344, \mathrm{p}<0.001)$, combinación $(\AA=.174$ $\mathrm{t}=2.558, \quad \mathrm{p}<0.001) \quad$ e internalización $(\AA=.280, \quad t=3.904, \quad p<0.01) \quad$ mostraron una relación positiva significativa con la innovación. Sin embargo, aunque de forma individual la externalización se correlaciona significativamente con la innovación $(\AA=.711, t=13.240, p<0.001)$, en el modelo múltiple $(\AA=.083, t=1.227$, $\mathrm{p}>0.05$ ) esta variable es excluida (Tabla 3).

\section{Tabla 3}

\section{Coeficientes de regresión sobre la innovación.}

\begin{tabular}{|c|c|c|c|c|c|c|}
\hline \multirow{2}{*}{$\begin{array}{l}\text { Variable } \\
\text { independiente }\end{array}$} & \multicolumn{2}{|c|}{ Modelo simple } & \multicolumn{2}{|c|}{ Modelo multivariado } & \multicolumn{2}{|c|}{ Diagnóstico de colinealidad } \\
\hline & $\beta$ & $\mathrm{t}$ & $\beta$ & $\mathrm{t}$ & Tolerancia & FIV \\
\hline Constante & & & 0.17 & 0.927 & & \\
\hline Socialización & 0.692 & $16.838^{* *}$ & 0.414 & $8.344^{\star *}$ & 0.452 & 2.210 \\
\hline Externalización & 0.711 & $13.240^{* *}$ & 0.083 & 1.227 & 0.307 & 3.253 \\
\hline Combinación & \multicolumn{2}{|c|}{$\begin{array}{c}0.707 \\
14.190^{* *} \\
0.174 \\
2.558^{* *}\end{array}$} & & & 0.286 & 3.491 \\
\hline Internalización & 0.85 & $12.165^{\star *}$ & 0.280 & $3.904^{* \star}$ & 0.423 & 2.364 \\
\hline R cuadrada & & & \multicolumn{2}{|c|}{0.793} & & \\
\hline R cuadrada corregida & & & \multicolumn{2}{|c|}{0.787} & & \\
\hline $\mathrm{F}$ & & & \multicolumn{2}{|c|}{$125.369^{* *}$} & & \\
\hline Durbin Watson & & & \multicolumn{2}{|c|}{1.601} & & \\
\hline
\end{tabular}

Nota: Coeficientes no estandarizados.

**Significativo al nivel de confianza de 0.001

Fuente: elaboración propia 
Las empresas crean nuevo conocimiento con fines de innovación a partir de obtener información valiosa del exterior (Cohen y Levinthal, 1994), que complementan con lo que ya conocen los miembros de la organización; en esta línea y siguiendo la propuesta de Nonaka y Takeuchi (1995) en las empresas estudiadas se produce una transformación del conocimiento tácito de los individuos en explícito a nivel grupal y organizacional.

Los resultados revelan que las empresas encuestadas realizan actividades de socialización, externalización, combinación e internalización que se relacionan positiva y significativamente con la innovación. Este resultado coincide con la investigación de Arceo et al. (2021) quienes demostraron que la innovación en las PYME catalanas es influenciada por las prácticas de GC que se llevan a cabo dentro de ellas, incluso estas prácticas son realizadas pese a no tenerse una conciencia plena de ello.

Este estudio demuestra que las pequeñas empresas socializan el conocimiento obtenido del exterior en pequeños grupos de trabajo, reuniones formales, reuniones informales y a través de la interacción que los vendedores realizan con los clientes, y que a veces realizan con la competencia, por ejemplo, cuando coinciden en la ferias comerciales o de emprendedores que organiza el gobierno.

La socialización del conocimiento es aprovechada por este grupo de empresas debido a su tamaño, al contar con una organización de pocos trabajadores, se logra una mejor integración. Coincidente con Plaza (2018) el hecho de concentrarse en un mismo centro de trabajo e interactuar permanentemente de manera informal, contribuye de manera importante a la socialización del conocimiento.

Sin embargo, falta por hacer. De acuerdo con Maldonado et al. (2012) para que las PYME puedan mejorar la GC, además, se requiere de la implementación de políticas y estrategias que permitan la adquisición de conocimiento externo, sobre todo de las instituciones públicas de educación superior y centros de investigación especializados en las PYME, así como mejorar la cultura organizacional para crear espacios propicios para socializar el conocimiento y la experiencia entre los empleados de la organización.

Con la externalización, el personal de las empresas encuestadas comparte experiencias, difunden información, aportan ideas, aclaran dudas y surgen otras, que posteriormente son investigadas. El grupo deduce posibles soluciones e identifica fallas en sus procedimientos. Detectan indicadores que les sirven para hacer comparaciones en su evolución. Aunque, coincidente con Maldonado et al. (2012) los resultados empíricos encontrados en este estudio denotan la necesidad de realizar una serie de actividades que generen un ambiente favorable para que los trabajadores de la organización puedan compartir aún más conocimientos, habilidades y experiencias con el resto de sus compañeros.

En la fase combinación, contrariamente a lo descubierto por Plaza (2018) en este grupo, las empresas se motivan a procesar la información que en colectivo han externalizado con el fin de difundir ese conocimiento al resto de la organización. Definen ideas formales, algunas rutinas, afectaciones a procesos y procedimientos; mismos que son retroalimentado entre el colectivo. El personal retoma la información y 
alimenta una base datos de sus clientes, competencia, novedades tecnológicas y dedican tiempo a diseñar rutinas, procedimientos y diagramas de flujo.

Con la internalización se vive el conocimiento combinado. A niveles más altos de conocimiento, competencias y habilidades de los empleados, se facilita la participación eficiente en tareas no solo rutinarias, sino rutinas con altos estándares de calidad aumentando así el valor agregado de la empresa (Simancas et al. 2018). Es importante señalar que algunas de estas empresas se encuentran certificadas por algún ISO, razón por la cual una vez internalizado el conocimiento, sus recientes experiencias los llevan a la revisión de sus manuales de procedimientos para incorporar las novedades o hacer los cambios correspondientes. Coincidente con Gutiérrez et al. (2015) en esta dimensión donde el conocimiento explicito se vuelve tácito, puede interpretarse que estas empresas alientan la experimentación a partir de las actividades que realizan en la dimensión combinación, de esta forma los resultados obtenidos se dirigen al mejoramiento o nuevos diseños de procedimientos, planes y estrategias que respondan a acciones de los competidores y a la satisfacción de gustos y preferencias de los clientes, entre otras.

De forma coincidente con Gutiérrez et al. (2015) se observan diferencias en la aportación que cada dimensión hace a la GC, para este caso la dimensión que presenta valores más bajos es la socialización y la dimensión más fortalecida es la internalización (diagrama 1). El ideal sería que las cuatro dimensiones presentaran valores altos, sin embargo, una aportación que hace este trabajo, es precisamente mostrar el desempeño real de la GC de las PYME encuestadas como un preámbulo a cambios importantes al interior y exterior de la organización, de tal manera que adquirir y compartir conocimiento y experiencias sean el despunte para enriquecer y mejorar los procesos de la innovación, y a su vez, lograr tanto un mayor valor añadido a los productos como incrementar el nivel del rendimiento empresarial (García et al. 2019).

Asimismo, se busca un modelo que compruebe una relación positiva y significativa, donde las cuatro dimensiones de la espiral del conocimiento se integren y revelen la secuencia de las actividades de cada fase, tratando de lograr la dinámica de la espiral, aunque, los resultados revelan que la dimensión externalización no es una variable predictora del modelo. Lo cual lleva a pensar que en la fase de socialización, también se realizan actividades de externalización como involucrar el intercambio de experiencias y difundir información. Debido al tamaño y a la falta de formalización de estas empresas, se advierte cierta dificultad para lograr una separación más precisa de actividades que indiquen dónde y cuándo termina la socialización e inicia la externalización. Con respecto a la combinación y la internalización si existe evidencia de documentación y formalización de actividades que indican esa separación entre las dimensiones.

Arantes et al. (2021) en su estudio sobre madurez de la GC muestran que las empresas dedican mayores esfuerzos a la socialización y la externalización y menores esfuerzos a la internalización y combinación. El grado de madurez que encuentran desarrollado en las PYME lo catalogan como "media" a diferencia de la categoría madurez "media-alta" que desarrollan las grandes 
empresas para las dimensiones de socialización y externalización y "media" para las dimensiones de combinación e internalización. Sin embargo, es necesario mejorar la gestión para todos los tamaños de empresas y dirigir esfuerzos a cada dimensión de GC. Las empresas necesitan potencializar los esfuerzos para brindar a su personal el desarrollo continuo de competencias, la promoción y el intercambio de conocimientos, que deben estar integrados en la cultura organizacional para impulsar su desempeño (Arantes et al. 2021).

Finalmente de acuerdo con Maldonado et al. (2012) la GC es una herramienta que se relaciona con la innovación que conduce a las PYME a la competitividad. Para ello es importante que las PYME sigan impulsando el desarrollo de actividades relacionadas con la GC, ya sea proporcionando a su trabajadores una formación enfocada a gestionar conocimiento, monitoreo constante del ambiente externo para extraer conocimiento novedoso o también, de ser posible, dirigir ciertos recursos en organizaciones ex profeso para que les ayuden a lograrlo. En suma, de acuerdo a Lloyd et al. (2002) las PYME deben aprovechar las ventajas que aporta su tamaño al desarrollo de la innovación. La flexibilidad en sus procesos y la adaptabilidad estructural, les ayudan a aprovechar los rápidos avances tecnológicos para su supervivencia y competitividad (Renuka y Venkateshwara, 2006).

Para que las empresas accedan a un mercado competitivo y globalizado como el actual, además de dirigir sus esfuerzos a la innovación tradicional, tienen la opción de incurrir en prácticas de innovación abierta de entrada y salida, que no solamente les ayudará a incrementar sus actividades de innovación, sino también su nivel de rendimiento financiero (García et al. 2019).

\section{Conclusiones}

Con el objetivo de la investigación se logró identificar que las dimensiones de la GC: socialización, externalización, combinación e internalización se relacionan positivamente con la innovación en las pequeñas empresas.

En la exploración individual de cada dimensión, se percibe la socialización del conocimiento como la dimensión menos valorada y la internalización aporta el puntaje más alto. En la conformación de un modelo multivariado que intenta concebir las cuatro dimensiones como el proceso de espiral que gestiona el conocimiento, contrariamente a lo que se esperaba, la externalización no es una variable predictora. Ante este resultado se espera que las PYME incursiones aún más en todas las etapas de la GC.

Los resultados indican que si las PYME quieren mejorar su nivel de crecimiento por medio de la innovación, tendrán que implementar estrategias idóneas para gestionar adecuadamente tanto el conocimiento tácito como el explícito existente en los trabajadores de la organización, sin olvidar la imperiosa necesidad de posicionarse a la expectativa del nuevo conocimiento que emana del exterior, así como también idear actividades que promulguen el adaptamiento a una cultura que cree espacios propicios para el intercambio de conocimiento y experiencias entre los empleados de la organización.

El estudio presenta una posible limitación respecto a la consideración de empresas pertenecientes solo al sector de manufactura, habría que continuar el 
estudio con otro sector y observar si el comportamiento es el mismo o si existen diferencias e incursionar en ellas para buscar una explicación. Así también, es conveniente dirigir la encuesta a las percepciones del resto de los empleados y cruzar información sobre las diferentes apreciaciones.

\section{Referencias bibliográficas}

Arantes, L. S., Martinelli Jr. O., Viegas, T. O. C. \& Rohenkoh, J. E. (2021). Maturity and level of knowledge management in the company: an application of Nonaka and Takeuchi model and Fuzzy Logic. Gestão \& Produção, 28(2), e5305. https://doi.org/10.1590/1806-96492020v28e5305

Arceo, M. G., Ramos, M. E. \& Acosta, de la C. J. T. (2021). Explorando las relaciones entre la gestión del conocimiento y las tecnologías de información con la innovación. Brazilian Journal of Business, 3(2), 1552-1566. https://doi.org/10.34140/ bjbv3n2-018

Bowerman, B. L., O'Connell, R. T. \& Koehler, A. B. (2007). Pronósticos, series de tiempo y regresión: un enfoque aplicado. 4a Ed. Cengage Learning.

Bueno, E. (2005). Fundamentos epistemológicos de dirección del conocimiento organizativo: desarrollo, medición y gestión de intangibles. Economía Industrial, (357), 13-26.

Chauvet, V. (2014). Absorptive capacity: Scale development and implications for future research. Management International, 19(1), 113-129. https:// doi.org/10.7202/1028493ar

Cohen, W. \& Levinthal, D. (1990). Absorptive capacity: A new perspective on learning and innovation. Administrative Sciences Quarterly, 35(1), 128-152. https://doi. org/10.2307/2393553

Cohen, W. \& Levinthal, D. (1994). Fortune favors the prepared firm. Management Science, 40, 227251. https://dx.doi.org/10.1287/ mnsc.40.2.227

De Jong, J. \& Vermeulen, P. (2006). Determinants of Product Innovation in Small Firms: A Comparison Across industries. International Small Business Journal, 25(6), 587-609.

Demuner, F. M. R. (2011). PYME competitivas. Eae Editorial Academia Española.

Demuner, F. M. R., Nava, R. R. M. y Sandoval, A. R. (2015). TI en la Dinámica del Conocimiento Empresarial. Universidad Autónoma del Estado de México. México. https://ri.uaemex.mx/ handle/20.500.11799/21798

Diario Oficial de la Federación. (2015). 30 diciembre.

Fernández, J. C. M. (2012) Determinantes de la capacidad de innovación en PYMES regionales. Documentos de traballo do Departamento de Economía Aplicada, (2). https:// dialnet.unirioja.es/servlet/ articulo?codigo $=4040071$

Fernhaber, S. \& Patel, P. (2012). How do young firms manage product portfolio complexity? The role of absorptive capacity and ambidexterity. Strategic Management Journal, 33(13), 1516-1539. https://www.jstor.org/ stable/41679865

Flor, M., Oltra, M. y García, C. (2011). La relación entre la capacidad de absorción del conocimiento externo y la estrategia empresarial: Un análisis exploratorio. Revista Europea de 
Demuner Flores, María del Rosario

Gestión del conocimiento en la innovación en pequeñas empresas de manufactura

Dirección y Economía de la Empresa, 20(1), 69-88.

García, V. M. Y., Maldonado, G. G. y Alvarado, C. A. (2019). Innovación abierta y rendimiento financiero en la industria manufacturera de México. Revista Venezolana de Gerencia, 24(87), 826-842. http://www.redalyc. org/articulo.oa?id=29060499012

González, M. J. J. y Álvarez, C. L. (2019). GestióndeConocimientoelnnovación Abierta: hacia la conformación de un modelo teórico relacional. Revista Venezolana de Gerencia, 24(88). $\quad$ https://www.redalyc.org/ jatsRepo/290/29062051013/html/ index.html

Gopalakrishnan, S. \& Damanpour, F. (1997). A review of innovation research in economics, sociology and technology management. Omega, 25(1), 15-28. https://www. sciencedirect.com/science/article/pii/ S0305-0483(96)00043-6

Gutiérrez, D. M. C., Sapién, A. A. L. y Piñón, H. L. C. (2015). Gestión de conocimiento en PYME del sector servicios en la ciudad de Chihuahua. Nova Scientia, 7(15). https://bit. ly/3dqiSVB

Hair, J., Black, W., Babin, B. \& Anderson, R. (2010). Multivariate data analysis. 7ma ed. Harlow: Pearson.

Índice Municipal de Actividad Económica IMAE. (2019). Índice Municipal de Actividad Económica.

Instituto Nacional de Estadística y Geografía INEGI. (2015). Censos económicos 2014. México. https:// www.inegi.org.mx

Instituto Nacional de Estadística y Geografía INEGI. (2019). Censos económicos 2018. Las empresas en los Estados Unidos Mexicanos. Censos Económicos 2019. México:
INEGI c2020. https://www.inegi.org. $\underline{\mathrm{mx}}$

Lloyd, R. L., Muller, K. \& Wall, S. (2002). Innovation and Educational Policy in SMEs: A Czech Perspective. Education \& Training, 44(8-9), 378-387. https://doi. org/10.1108/00400910210449213

Maldonado, G. G., Martínez, S. M. C. y García, R. R. (2012). Gestión del conocimiento y crecimiento en la PYME manufacturera de Aguascalientes (México). Cuadernos de Administración, 28(47), 25-36. http://www.redalyc.org/articulo. oa?id=225025086003

Manual de Oslo. (2018). The Measurement of Scientific Technological and Innovation Activities. Guidelines for collecting, reporting and using data innovation. OCDE, EUROSTAT, $4^{\circ} \mathrm{Ed}$.

Medina, G. V. (2017). Gestión del conocimiento y capital social: su relación en contextos universitarios. Revista Cubana de Información en Ciencias de la Salud, 28(3), 23072113. https://www.medigraphic.com/ pdfs/acimed/aci-2017/aci173f.pdf

Milton, S. y Arnold, J. (2004). Probabilidad y Estadística con Aplicaciones para Ingeniería y Ciencias del Comportamiento. McGraw Hill.

Nieto, M. \& Quevedo P. (2005). Absorptive capacity, technological opportunity, knowledge spillovers, and innovative effort. Technovation, 25, 1141$1157 . \quad$ https://doi.org/10.1016/j. technovation.2004.05.001

Nonaka, I. \& Takeuchi, H. (1995). The knowledge- creating company: How Japanese companies create the dynamics of innovation. Oxford University Press.

Olea, M. J., Contreras, O. F. y Barcelo, 
V. M. (2016). Las capacidades de absorción del conocimiento como ventajas competitivas paralainserción de PYME en cadenas globales de valor. Estudios Gerenciales, 32(139), 127-136. https://doi.org/10.1016/j. estger.2016.04.002

Pardo, A. y Ruíz, M. A. (2002). SPSS 11. Guía para el análisis de datos. McGraw Hill.

Pérez, M. M. (2016), Gestión del conocimiento: Orígenes y evolución. El Profesional de la Información, 25(4), 526-534. https://doi. org/10.3145/epi.2016.jul.02

Plaza, A. J. J. (2018). Creación de conocimiento en pequeños establecimientos hoteleros de España. Revista Venezolana de Gerencia, 23(82), 1-15. http://www.redalyc.org/articulo. oa?id=29056115003

Porter, M. E. (1990). The competitive advantage of nations. Harvard Business Review, 68(2), 73-93. http://secure.com.sg/courses//Cl/ Grab/Reading Articles/L04_A03 Porter.pdf

Quiñonez Zuñiga, C., y Rivera Martínez, W. (2021). Modelo de gestión del conocimiento para centros de productividad e innovación. Telos: revista de Estudios Interdisciplinarios en Ciencias Sociales, 23(2), 347-366. www.doi.org/10.36390/telos232.09

Renuka, S. D. \& Venkateshwara, B. A. A. (2006). Comparative Study of Human Resource Management Practices and Advanced Technology Adoption of SMEs with and without ISO Certification. Singapore Management Review, 28(1), 41- 60.
Restrepo, M. J. A., Loaiza, Q. O. L. y Galvez, A. E. (2015). Innnovación y desempeño de las micro, pequeñas y medianas empresas en Colombia. Revista de Ciencias Sociales, XXII(4), 24-40. https://www.redalyc. org/pdf/280/28056724003.pdf

Simancas Trujillo, R. A., Silvera Sarmiento, A. de J., Silvera Sarmiento, A. de J., Garcés Giraldo, L. F., Garcés Giraldo, L. F., Hernández Palma, H. G., \& Hernández Palma, H. G. (2018). Administración del capital humano: factor de productividad empresarial en pequeñas y medianas empresas de Barranquilla. Revista Venezolana De Gerencia, 23(82), 377-391. https://doi.org/10.37960/ revista.v23i82.23754

Tabachnick, B. \& Fidell, L. (2013). Using multivariate statistics. 6ta ed. Pearson.

Tarling, R. (2009). Statistical modelling for social researchers. Routledge.

Teece, D. T. (2016). Dynamic capabilities and entrepreneurial management in large organizations: Toward a theory of the entrepreneurial firms. European Economic Review, 86, 202-216. https://doi.org/10.1016/j. euroecorev.2015.11.006

Wang, M. \& Yang, T. (2016). Investigating the success of knowledge management: An empirical study of small and medium-sized enterprises. Asia Pacific Management Review, 21(2), 79-91. http://dx.doi. org/10.1016/j.apmrv.2015.12.003.

Zahra, S. \& George, G. (2002). Absorptive capacity: A Review and reconceptualization. Academy of Management Review, 27(2), 185203. https://doi.org/10.2307/4134351 